PROCEEDINGS OF THE

AMERICAN MATHEMATICAL SOCIETY

Volume 136, Number 10, October 2008, Pages 3709-3716

S 0002-9939(08)09413-6

Article electronically published on May 7, 2008

\title{
CANONICAL COVERS AND DIMENSION OF Z-SETS IN THE HILBERT CUBE
}

\author{
E. CUCHILLO-IBÁÑEZ AND M. A. MORÓN
}

(Communicated by Alexander N. Dranishnikov)

\begin{abstract}
In this paper we characterize the finite dimensionality of a compact Z-set in the Hilbert cube in terms of the existence of a particular canonical cover in its complement.
\end{abstract}

\section{INTRODUCTION}

In [1] it was suggested that topological invariants of compact Z-sets of the Hilbert cube, $Q$, could be described in terms of metric-uniform invariants of their complements. In particular it was asked how the dimension can be described in this context. Later, in [4] it was proved that the topological category for compact Zsets of the Hilbert cube is isomorphic to the $C_{0}$-coarse geometry category of their complements. The $C_{0}$-coarse structure of a metric space was introduced by Wright 11 (see also [12]). We recommend 10 for the basic definitions of general coarse spaces.

One of the consequences of [4] is that the complements of two compact Z-sets are $C_{0}$-coarsely equivalent if and only if those complements are uniformly homeomorphic (with respect to the metrics induced by that fixed on the Hilbert cube) and it is equivalent to the fact that the two compact Z-sets are homeomorphic.

In view of the equivalence of categories described in [4, it is natural to consider the problem of describing topological invariants of Z-sets in terms of $C_{0}$-coarse invariants of their complements. In this way the asymptotic dimension of such a coarse structure on the complement appears to be an appropriate tool for describing, from the outside, the topological dimension of a Z-set. Indeed in 44 it was proved that the dimension of a Z-set can be interpreted in terms of the asymptotic dimension of the $C_{0}$-coarse structure on its complement. See [10] for the definition of asymptotic dimension of a coarse space.

Instead of the $C_{0}$-asymptotic dimension, in this paper we use the well-known concept of canonical covers, used by Dugundji in [5, to characterize the finitedimensionality of a Z-set or, equivalently, the finiteness of the asymptotic dimension of the $C_{0}$-coarse structure in its complement. We then point out that although the tools used in the definition of canonical cover are topological in nature, in fact this concept is in the core of the $C_{0}$-coarse geometry.

Received by the editors October 12, 2006, and, in revised form, August 20, 2007.

2000 Mathematics Subject Classification. Primary 54F45, 52B11; Secondary 54E15.

Key words and phrases. Compact Z-set, canonical cover, nerve of a cover, covering dimension. The authors were supported by the MEC, MTM2006-0825.

(C)2008 American Mathematical Society
Reverts to public domain 28 years from publication 3709 
First we show how to project to the outside certain cofinal sequences of open covers of the Z-set, hence constructing canonical covers of its complements.

Later, in the usual way, we replace this complement by a countable locally finite polyhedron where a topological copy of the Z-set can be viewed as its end space. This construction is an extension of the fact that 0 -dimensional metrizable compacta can be represented as the end space of a locally finite simplicial tree.

The general result we obtain is that a compact Z-set is finite-dimensional if and only if its complement admits a canonical cover of finite order. We also relate the dimension with the order of the canonical cover. Kroonenberg 9 obtained some dual related results. In particular she studied, in terms of the complement, when a finite dimensional closed subset of the Hilbert cube is a Z-set.

For the reader's convenience we recommend 3 for the study of Z-sets; see also 6. 8] for dimension theory and [10] for a global view of coarse geometry concepts quoted, but not used, in this paper.

The authors are very grateful to the referee whose suggestions improved significantly an earlier version of this paper.

\section{CANONICAL COVERS AND DIMENSION From the OUtSidE}

Our final intention is to interpret the inequality $\operatorname{dim} X \leq n$ in terms of $Q \backslash X$, where $X \subset Q$ is a compact Z-set in the Hilbert cube $Q$. Recall that a closed subset $A$ in the Hilbert cube $(Q, d)$ is said to be a Z-set in $Q$ provided that for every $\epsilon>0$ there exists a continuous map $f: Q \longrightarrow Q \backslash A$ such that $d(f(p), p)<\epsilon$ for each $p \in Q$. Moreover, any compact metric space can be embedded in the Hilbert cube as a Z-set.

Definition 2.1. Let $X$ be a topological space and $A$ a closed subset of $X$. A canonical cover of $X \backslash A$ in $X, \gamma$, is an open cover of $X \backslash A$ such that:

i) $\gamma$ is a locally finite family, and

ii) for each neighborhood $V$, in $X$, of a point $a \in A$ there exists another neighborhood $W$ of $a$ in $X, W \subset V$, such that every element $U \in \gamma$ which meets $W$ is contained in $V$.

This concept was used by Dugundji [5] to obtain his famous generalization of Tietze's Extension Theorem. As Antonyan noted in 2, the second condition implies that every neighborhood of any boundary point of $A$ in $X$ contains infinitely many open sets in $\gamma$. This was a requisite to be a canonical cover in its original definition (see [5]). Other useful results obtained using these techniques can be found in the book [7].

To treat the dimension from the outside, that is from the complement of a Z-set, we need some preliminary results:

Proposition 2.2. Let $X$ be a compact subset of $Q$. For each canonical cover $\gamma$ of $Q \backslash X$ and every open set $G \subset Q$, with $X \subset G$, the family of sets of $\gamma$ that have empty intersection with $G$ is finite.

Proof. Assume that there exists a sequence $\left\{U_{n}\right\}_{n \in \mathbb{N}}$ of different elements of $\gamma$ such that, for every $n \in \mathbb{N}, U_{n} \cap G=\varnothing$. Consider a sequence $\left\{x_{n}\right\}_{n \in \mathbb{N}}$ of points such that $x_{n} \in U_{n}$. As this sequence is contained in the compact $Q \backslash G$, the sequence has an accumulation point $z_{0} \in Q \backslash G \subset Q \backslash X$. In this way, every neighborhood of $z_{0}$ intersects an infinite number of members of the canonical cover $\gamma$, which is not possible. 
Similar arguments as those in the above proposition lead us to the following

Proposition 2.3. Let $X$ be a compact subset of $Q$. For each canonical cover $\gamma$ of $Q \backslash X$ and every open set $G \subset Q$ such that $X \subset G$, the family of sets of $\gamma$ that are not contained in $G$ is finite.

Remark 2.4. Note that the results above say that the canonical covers in $Q \backslash X$ are in fact uniform covers (see Definition 5.29 in [10]) for the $C_{0}$-coarse structure in $Q \backslash X$.

Another property we will use is

Proposition 2.5. Let $X$ be a compact subset of $Q$. Each member of a canonical cover $\gamma$ of $Q \backslash X$ intersects only a finite family of elements of $\gamma$.

Proof. Let $U_{0} \in \gamma$. As $\gamma$ is a canonical cover of $Q \backslash X, \overline{U_{0}} \cap X=\varnothing$, where $\overline{U_{0}}$ means the closure of $U_{0}$ in $Q$.

Define $G=Q \backslash \overline{U_{0}}$. Observe that $G$ is open in $Q$ and $X \subset G$. As every $U \in \gamma$ that intersects $U_{0}$ satisfies $U \nsubseteq G$, from Proposition 2.3 they are a finite collection.

To fix the notation we choose the following definition of order.

Definition 2.6. Let $X$ be a set and $\mathcal{A}$ a family of subsets of $X$. By the order of the family $\mathcal{A}$, ord $\mathcal{A}$, it means the largest integer $n$ such that there are $n+1$ members of the family $\mathcal{A}$ which have a non-empty intersection. If no such integer exists, we say that $\mathcal{A}$ has order $\infty$.

Proposition 2.7. Let $X$ be a compact subspace of the Hilbert cube $(Q, d)$ and $\mathcal{U}=\left\{U_{i}\right\}_{i=1}^{m}$ be a finite open cover of $X$ such that $\operatorname{ord}(\mathcal{U}) \leq n$. For every $\varepsilon>0$ there exists a family (an $\varepsilon$-envelopment) $\mathcal{W}=\left\{W_{i}\right\}_{i=1}^{m}$ of open subsets of $Q$ that satisfies:

a) $W_{i} \cap X=U_{i}$ for each $i \in\{1, \ldots, m\}$.

b) $W_{i} \subset B\left(U_{i}, \varepsilon\right)$ and, so, $\operatorname{diam}\left(W_{i}\right) \leq \operatorname{diam}\left(U_{i}\right)+2 \varepsilon$ for each $i \in\{1, \ldots, m\}$.

c) For every subset $\Lambda \subset\{1, \ldots, m\}, \bigcap_{r \in \Lambda} W_{r} \neq \varnothing$ if and only if $\bigcap_{r \in \Lambda} U_{r} \neq \varnothing$. In particular $\operatorname{ord}(\mathcal{W})=\operatorname{ord}(\mathcal{U})$.

Proof. Choose an index $i \in\{1, \ldots, m\}$. If $U_{i}=X$, take $W_{i}=B\left(X, \frac{\varepsilon}{2}\right)$. In the general case, $U_{i} \neq X$, for every $x \in U_{i}$ consider

$$
\varepsilon_{x, i}=\frac{1}{2} \min \left\{d\left(x, X \backslash U_{i}\right), \varepsilon\right\}
$$

Clearly $B\left(x, \varepsilon_{x, i}\right) \cap X \subset U_{i}$. Define

$$
W_{i}=\bigcup_{x \in U_{i}} B\left(x, \varepsilon_{x, i}\right) .
$$

By its construction,

$$
W_{i} \cap X=U_{i} \quad \text { and } \quad W_{i} \subset B\left(U_{i}, \frac{\varepsilon}{2}\right) .
$$

When $U_{j_{1}} \cap \cdots \cap U_{j_{k}} \neq \varnothing, j_{1}, \ldots, j_{k} \in\{1, \ldots, m\}$, then $W_{j_{1}} \cap \cdots \cap W_{j_{k}} \neq \varnothing$. Reciprocally, if $W_{j_{1}} \cap \cdots \cap W_{j_{k}} \neq \varnothing$ and $U_{j_{r}} \neq X$ for all $r \in\{1, \ldots, k\}$ (the other option is reducible to this one), let $z \in W_{j_{1}} \cap \cdots \cap W_{j_{k}}$. There exist $x_{1} \in$ $U_{j_{1}}, \ldots, x_{k} \in U_{j_{k}}$ such that

$$
z \in B\left(x_{1}, \varepsilon_{x_{1}, j_{1}}\right) \cap \cdots \cap B\left(x_{k}, \varepsilon_{x_{k}, j_{k}}\right) .
$$


Suppose that $s \in\{1, \ldots, k\}$ satisfies

$$
\varepsilon_{x_{s}, j_{s}}=\min \left\{\varepsilon_{x_{r}, j_{r}}: r \in\{1, \ldots, k\}\right\} .
$$

For all $r \in\{1, \ldots, k\}, r \neq s$, we have

$$
d\left(x_{r}, x_{s}\right) \leq d\left(x_{r}, z\right)+d\left(z, x_{s}\right)<\varepsilon_{x_{r}, j_{r}}+\varepsilon_{x_{s}, j_{s}} \leq 2 \varepsilon_{x_{r}, j_{r}} .
$$

From the definition of $\varepsilon_{x_{r}, j_{r}}$, we have that $x_{s} \in U_{j_{r}}$ for every $r \in\{1, \ldots, k\}$. In such a way,

$$
x_{s} \in U_{j_{1}} \cap \cdots \cap U_{j_{k}} \neq \varnothing .
$$

Finally, we conclude that

$$
\operatorname{ord}\left(\left\{W_{i}\right\}_{i=1}^{m}\right)=\operatorname{ord}\left(\left\{U_{i}\right\}_{i=1}^{m}\right) .
$$

Remark 2.8. Open sets of $Q$ belonging to envelopments (constructed as in the preceeding proposition) of different open covers of $X$ have a non-empty intersection if and only if the original members of the open covers of $X$ also have a non-empty intersection.

Proposition 2.9. Consider the Hilbert cube with a fixed metric $(Q, d)$. Let $X \subset Q$ be a compact subset with $\operatorname{dim}(X)=n$. Then there is a canonical cover $\gamma$ of $Q \backslash X$ with $\operatorname{ord}(\gamma) \leq 2 n+1$.

Proof. Consider a sequence $\left\{\mathcal{U}_{m}, m \in \mathbb{N}\right\}$ of finite open coverings of $X$ with the following properties:

1. $\mathcal{U}_{1}=\{X\}$ and $\mathcal{U}_{m+1} \geq \mathcal{U}_{m}$ for every $m \in \mathbb{N}$. That is, $\mathcal{U}_{m+1}$ is a refinement of $\mathcal{U}_{m}$.

2. $\lim _{m \rightarrow \infty} \operatorname{mesh}\left(\mathcal{U}_{m}\right)=0$, where mesh represents the maximum of the diameters of all elements in $\mathcal{U}_{m}$ with respect to the inherit metric, and the sequence $\left\{\operatorname{mesh}\left(\mathcal{U}_{m}\right)\right\}_{m \in \mathbb{N}}$ is strictly decreasing.

3. $\operatorname{ord}\left(\mathcal{U}_{m}\right) \leq n$ for every $m \in \mathbb{N}$.

For each $m \in \mathbb{N}$, take a $1 / m$-envelopment $\mathcal{W}_{m}=\left\{W_{m, j}\right\}_{j=1}^{k_{m}}$ of the cover $\mathcal{U}_{m}=$ $\left\{U_{m, j}\right\}_{j=1}^{k_{m}}$ as described in the above proposition. We can suppose that if $B_{m}=$ $\bigcup_{j=1}^{k_{m}} W_{m, j}$, then $\left\{B_{m}, m \in \mathbb{N}\right\}$ is a base of open neighborhoods of $X$ in $Q$ such that $\bar{B}_{m+1} \subset B_{m}$ for every $m \in \mathbb{N}$ and that $\bar{B}_{2} \neq Q$. Define

$$
\begin{gathered}
\beta_{1,1}=Q \backslash \bar{B}_{2}, \\
\beta_{m}=\left\{\beta_{m, j}=W_{m-1, j} \backslash \bar{B}_{m+1}, j=1, \ldots, k_{m-1}\right\} \text { for } m \geq 2 .
\end{gathered}
$$

We will show that the family

$$
\gamma=\left\{\beta_{p, i}: p \in \mathbb{N}, i=1, \ldots, k_{p}\right\}
$$

is a canonical cover of $Q \backslash X$, where $k_{1}=1$. In fact, it is clear that $\gamma$ is an open cover of $Q \backslash X$. Since the families $\beta_{m}$ are finite sets, $\left\{B_{m}, m \in \mathbb{N}\right\}$ is a base of open neighborhoods of $X$ and, for all $m, \bar{B}_{m+1} \subset B_{m}$ and $\beta_{m, j} \cap \bar{B}_{m+1}=\varnothing$, $j \in\left\{1, \ldots, k_{m-1}\right\}$, then $\gamma$ is a locally finite family. Given $x_{0} \in X$ and an open neighborhood of $x_{0}$ in $Q, A_{x_{0}}$, from the properties of the envelopments $\mathcal{W}_{m}$ there exists $m_{0} \in \mathbb{N}$ which is the minimum of the numbers $m \in \mathbb{N}$ which satisfy the following property: "if $x_{0} \in W_{m, j}$, then $W_{m, j} \subset A_{x_{0}}$ ". If we choose $V_{x_{0}}$ equal to any of the $W_{p, j}$, for $p \geq m_{0}+2$, containing $x_{0}$, it is easy to verify that if an element of $\gamma$ meets $V_{x_{0}}$, then it is contained in $A_{x_{0}}$.

Finally, ord $(\gamma) \leq 2 n+1$, because for each $p$ there are at most $n+1$ elements of $\beta_{p}$ with a non-empty intersection and if $\beta_{p, i} \bigcap \beta_{l, j} \neq \varnothing$, necessarily $|p-l| \leq 1$. 
Remark 2.10. Note that in the above result there is a gap between the dimension of the Z-set, $n$, and the order of the constructed canonical cover, $2 n+1$. The gap is just $n+1$. In the case $n=0$, this gap must be at least 1 . Moreover, using the fact that the family of partitions is cofinal for finite open covers in 0-dimensional compacta, the above construction can be refined to obtain the classical result that those spaces are just the end spaces of locally finite simplicial trees.

Can we always find a canonical cover with gap 1? In a work in progress we are studying this question, but until now we have only been able to prove that the answer to this question is affirmative provided $n=0,1$.

In order to obtain a reciprocal result we first note:

Let $\gamma$ be a canonical cover of $Q \backslash X, \mathcal{N}(\gamma)$ the nerve of $\gamma$, and consider the space $\mathcal{N}(\gamma) \cup X$ topologized as it is indicated in [5, Theorem 3.1] (see also 7, Proposition II.12.1]). Specifically, a basis of open sets for this topology is given by

- the open subsets of $\mathcal{N}(\gamma)$ endowed with the Whitehead topology and

- the subsets $V^{*}$ constructed as follows: for each point $x \in X$ and each open neighborhood $V$ of $x$ in $Q$, the set $V^{*}$ in $\mathcal{N}(\gamma) \cup X$ consists of the points of $X \cap V$ and those of the stars of every vertex of $\mathcal{N}(\gamma)$ corresponding to a set of the covering $\gamma$ contained in $V$.

We denote by

$$
\pi: Q \longrightarrow \mathcal{N}(\gamma) \cup X
$$

the continuous map built in [5, Theorem 3.1] which is the identity, and a homeomorphism, over $X$.

In this case we obtain

Proposition 2.11. The space $\mathcal{N}(\gamma) \cup X$ is compact and metrizable.

Proof. Let $\mathcal{G}=\left\{G_{\lambda}\right\}_{\lambda \in \Lambda}$ be an open cover of $\mathcal{N}(\gamma) \cup X$.

Since $X$ is compact, there exists a finite number of elements of $\mathcal{G}$ which cover $X$,

$$
X \subset G_{1} \cup \cdots \cup G_{m},
$$

and there exists a finite number of basic open sets $V_{1}^{*}, \ldots, V_{n}^{*}$ such that

$$
X \subset V_{1}^{*} \cup \cdots \cup V_{n}^{*} \subset G_{1} \cup \cdots \cup G_{m} .
$$

As

$$
(\mathcal{N}(\gamma) \cup X) \backslash\left(G_{1} \cup \cdots \cup G_{m}\right) \subset(\mathcal{N}(\gamma) \cup X) \backslash\left(V_{1}^{*} \cup \cdots \cup V_{n}^{*}\right) \subset \mathcal{N}(\gamma),
$$

it suffices to see that the closed subset

$$
(\mathcal{N}(\gamma) \cup X) \backslash\left(V_{1}^{*} \cup \cdots \cup V_{n}^{*}\right)=\mathcal{N}(\gamma) \backslash\left(V_{1}^{*} \cup \cdots \cup V_{n}^{*}\right)
$$

is compact. Following their definition, for each $i \in\{1, \ldots, n\}$,

$$
V_{i}^{*}=\pi\left(V_{i} \cap X\right) \cup \bigcup_{U \in \gamma, U \subset V_{i}} S t\left(v_{U}\right),
$$

where $V_{i}$ is an open set in $Q, v_{U}$ is the vertex in $\mathcal{N}(\gamma)$ corresponding to $U \in \gamma$ and $\operatorname{St}\left(v_{U}\right)$ is the star of $v_{U}$ in the infinite polyhedron $\mathcal{N}(\gamma)$. Clearly

$$
X \subset V_{1} \cup \cdots \cup V_{n} .
$$

We will show there are a finite number of vertices of $\mathcal{N}(\gamma)$ in $\mathcal{N}(\gamma) \backslash\left(V_{1}^{*} \cup \cdots \cup\right.$ $\left.V_{n}^{*}\right)$. In fact, since $X$ is compact and $\gamma$ is a canonical cover of $Q \backslash X$, there exist $W_{1}, \ldots, W_{l}$, open subsets of $Q$, such that they have a non-empty intersection with 
$X$, they cover $X$ and if $U \in \gamma$ intersects some of them, then $U$ is contained in $V_{i}$ for certain $i \in\{1, \ldots, n\}$. Take $U_{0} \in \gamma$ and its associated vertex $v_{U_{0}} \in \mathcal{N}(\gamma)$. The following are equivalent:

i) $v_{U_{0}} \notin V_{1}^{*} \cup \cdots \cup V_{n}^{*}$.

ii) For all $i \in\{1, \ldots, n\}, v_{U_{0}} \notin \bigcup_{U \in \gamma, U \subset V_{i}} S t\left(v_{U}\right)$.

iii) For every $i \in\{1, \ldots, n\}, U_{0} \nsubseteq V_{i}$.

Hence, when $v_{U_{0}} \notin V_{1}^{*} \cup \cdots \cup V_{n}^{*}$, then

$$
U_{0} \cap\left(W_{1} \cup \cdots \cup W_{l}\right)=\varnothing .
$$

In this manner, as $W_{1} \cup \cdots \cup W_{l}$ is an open set of $Q$ that contains $X$, by Proposition 2.2 there is a finite collection of elements of the canonical cover $\gamma$ which have an empty intersection with $W_{1} \cup \cdots \cup W_{l}$. Therefore, in $\mathcal{N}(\gamma) \backslash\left(V_{1}^{*} \cup \cdots \cup V_{n}^{*}\right)$ there is a finite number of vertices of $\mathcal{N}(\gamma)$.

Now we classify the vertices of $\mathcal{N}(\gamma)$ into two classes:

a) Vertices that are not in $V_{1}^{*} \cup \cdots \cup V_{n}^{*}$. There are only a finite number of them: $v_{U_{1}}, \ldots, v_{U_{k}}$.

b) Vertices $v_{U}$ that are in $V_{1}^{*} \cup \cdots \cup V_{n}^{*}$, and, therefore, there exists $V_{i}, i \in$ $\{1, \ldots, n\}$, such that $U \subset V_{i}$.

Obviously, when all the vertices are considered:

$$
\mathcal{N}(\gamma)=\bigcup_{j=1}^{k} S t\left(v_{U_{j}}\right) \cup \bigcup_{i=1}^{n} \bigcup_{U \in \gamma, U \subset V_{i}} S t\left(v_{U}\right)
$$

So

$$
\mathcal{N}(\gamma) \backslash\left(V_{1}^{*} \cup \cdots \cup V_{n}^{*}\right)=\mathcal{N}(\gamma) \backslash \bigcup_{i=1}^{n} \bigcup_{U \in \gamma, U \subset V_{i}} S t\left(v_{U}\right) \subset \bigcup_{j=1}^{k} S t\left(v_{U_{j}}\right) \subset \bigcup_{j=1}^{k} \overline{S t}\left(v_{U_{j}}\right)
$$

where $\overline{S t}$ denotes the closed star.

Finally, due to Proposition 2.5, as each closed star is a finite polyhedron they are compact subspaces. Hence, the closed subset $\mathcal{N}(\gamma) \backslash\left(V_{1}^{*} \cup \cdots \cup V_{n}^{*}\right)$ is contained in a finite union of compact sets and it is also compact.

As was pointed out in [5] it is not difficult to verify that the space $\mathcal{N}(\gamma) \cup X$, with the mentioned topology, is a Hausdorff space. Thus, if we show that $\mathcal{N}(\gamma) \cup X$ is second countable then we obtain its metrizability. In fact, a countable base for its topology is the union of the following families of open subsets:

- $\left\{V_{n}^{*}\right\}_{n \in \mathbb{N}}$, where $\left\{V_{n}\right\}_{n \in \mathbb{N}}$ is the family of all elements in an open countable base of $Q$ meeting $X$.

- $\left\{W_{m, k} \mid m, k \in \mathbb{N}\right\}$, where, for each $m \in \mathbb{N},\left\{W_{m, k}\right\}_{k \in \mathbb{N}}$ is a countable open base of the interior (in $\mathcal{N}(\gamma)$ ) of the finite polyhedron contained in $\mathcal{N}(\gamma)$ determined by the members of the canonical cover that are not contained in $Q \backslash B\left(X, \frac{1}{m}\right)$.

Proposition 2.12. Let $X$ be a compact metric space embedded in the Hilbert cube $Q$ as a $Z$-set. If there exists a canonical cover of $Q \backslash X$ whose order is less than or equal to $m$, then $\operatorname{dim}(X) \leq m$.

Proof. If $\gamma$ is a canonical cover of $Q \backslash X$ whose order is less than or equal to $m$, the nerve of $\gamma, \mathcal{N}(\gamma)$, is an infinite polyhedron whose simplices have dimension less 
than or equal to $m$. Consider the mentioned extended canonical projection

$$
\pi:(Q, d) \longrightarrow\left(\mathcal{N}(\gamma) \cup X, d^{\prime}\right)
$$

where $d^{\prime}$ is a distance function which defines the topology of $\mathcal{N}(\gamma) \cup X$.

Now we are going to bound the dimension of the compact metric space $X$ via $\varepsilon$-mappings to polyhedra [6, Theorem 1.10.14]. Choose a number $\varepsilon>0$. As $\pi$ : $Q \rightarrow \mathcal{N}(\gamma) \cup X$ is a uniformly continuous map, take $\delta>0$ such that if $d\left(z_{1}, z_{2}\right)<\delta$, $z_{1}, z_{2} \in Q$, then $d^{\prime}\left(\pi\left(z_{1}\right), \pi\left(z_{2}\right)\right)<\varepsilon / 2$. Since $X$ is a Z-set, for that $\delta>0$ there exists a continuous map

$$
f_{\delta}: Q \longrightarrow Q
$$

which verifies

$$
f_{\delta}(Q) \cap X=\varnothing
$$

and

$$
d\left(x, f_{\delta}(x)\right)<\delta \text { for all } x \in Q .
$$

Define the map

$$
F_{\varepsilon}:\left(X, d^{\prime}\right) \longrightarrow \mathcal{N}(\gamma) \cup X
$$

as

$$
F_{\varepsilon}(t)=\left(\pi \circ f_{\delta} \circ\left(\left.\pi\right|_{X}\right)^{-1}\right)(t) .
$$

Observe that $F_{\varepsilon}(X) \subset \mathcal{N}(\gamma)$. Since $X$ is a compact space, $F_{\varepsilon}$ is a continuous map and $\mathcal{N}(\gamma)$ is an infinite polyhedron whose simplices have dimension less than or equal to $m$, the image of $F_{\varepsilon}$ is a compact space which, necessarily, is contained in a finite subpolyhedron of $\mathcal{N}(\gamma)$. So, the dimension of the image of $F_{\varepsilon}$ is less than or equal to $m$.

Finally, the diameter of all the fibres of $F_{\varepsilon}$ is less than $\varepsilon$. In fact, as the image of $F_{\varepsilon}$ lies in $\mathcal{N}(\gamma)$, take $y \in \mathcal{N}(\gamma)$ and $x_{1}, x_{2} \in X$ such that $F_{\varepsilon}\left(\pi\left(x_{1}\right)\right)=F_{\varepsilon}\left(\pi\left(x_{2}\right)\right)=y$ :

$$
\begin{aligned}
& d^{\prime}\left(\pi\left(x_{1}\right), \pi\left(x_{2}\right)\right) \leq d^{\prime}\left(\pi\left(x_{1}\right), \pi\left(f_{\delta}\left(x_{1}\right)\right)\right)+d^{\prime}\left(\pi\left(f_{\delta}\left(x_{1}\right)\right), \pi\left(x_{2}\right)\right) \\
& \quad=d^{\prime}\left(\pi\left(x_{1}\right), \pi\left(f_{\delta}\left(x_{1}\right)\right)\right)+d^{\prime}\left(\pi\left(f_{\delta}\left(x_{2}\right)\right), \pi\left(x_{2}\right)\right)<\frac{\varepsilon}{2}+\frac{\varepsilon}{2}=\varepsilon .
\end{aligned}
$$

Therefore,

$$
\operatorname{dim}(X) \leq m
$$

Using the results above and the equivalence of categories in 4 we have

Theorem 2.13. Consider the Hilbert cube with a fixed metric $(Q, d)$. Let $X \subset Q$ be a compact Z-set. Then the following sentences are equivalent:

1. $X$ is finite-dimensional.

2. The asymptotic dimension of the $C_{0}$-coarse structure induced by the metric $d$ in $Q \backslash X$ is finite.

3. $Q \backslash X$ admits a finite order canonical cover. 


\section{REFERENCES}

1. M. Alonso-Morón and A. González Gómez, The Hausdorff metric and classifications of compacta, Bull. London Math. Soc. 38 (2006), 314-322. MR2215924(2006m:54019)

2. S. Antonyan, A short proof that hyperspaces of Peano continua are absolute retracts, Proc. Amer. Math. Soc. 129 (2001), 2187-2189. MR1825932 (2002c:54004)

3. T.A. Chapman, Lectures on Hilbert Cube Manifolds, Amer. Math. Soc., Providence, RI, 1976. MR0423357 (54:11336)

4. E. Cuchillo-Ibáñez, J. Dydak, A. Koyama and M.A. Morón, $C_{0}$-coarse geometry of complements of Z-sets in the Hilbert cube, Transactions A.M.S. (to appear).

5. J. Dugundji, An extension of Tietze's theorem, Pacific Journ. Math. 1 (1951), 353-367. MR 0044116 (13:373c)

6. R. Engelking, Theory of Dimensions Finite and Infinite, Heldermann Verlag, Lemgo, 1995. MR 1363947 (97j:54033)

7. S.-T. Hu, Theory of retracts, Wayne State University Press, Detroit, MI, 1965. MR.0181977 $(31: 6202)$

8. W. Hurewicz and H. Wallman, Dimension Theory, Princeton University Press, Princeton, NJ, 1941. MR.0006493 (3:312b)

9. N. Kroonenberg, Characterization of finite-dimensional Z-sets, Proc. Amer. Math. Soc. 43 (1974), 421-427. MR0334221 (48:12540)

10. J. Roe, Lectures on Coarse Geometry, American Mathematical Society, Providence, RI, 2003. MR2007488 (2004g:53050)

11. N. Wright, $\mathrm{C}_{0}$ coarse geometry and scalar curvature, J. Funct. Anal. 197 (2003), 469-488. MR 1960422 (2003m:58034)

12. N. Wright, The coarse Baum-Connes conjecture via $\mathrm{C}_{0}$ coarse geometry, J. Funct. Anal. 220 (2005), 265-303. MR2119281(2006d:58023)

Departamento Matemática Aplicada, E.T.S.I. Montes, Universidad Politécnica, 28040 MADRID, SPAIN

E-mail address: eduardo.cuchillo@upm.es

Departamento Geometría y Topología, Facultad de Cc. Matemáticas, Universidad Complutense, 28040 Madrid, Spain

E-mail address: ma_moron@mat.ucm.es 